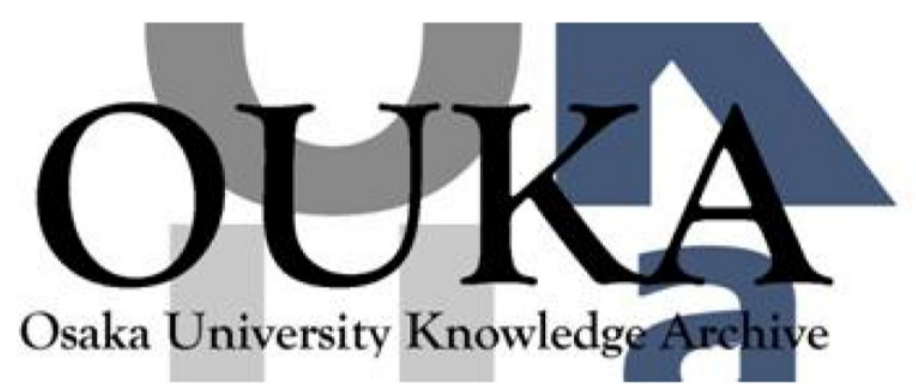

\begin{tabular}{|c|l|}
\hline Title & $\begin{array}{l}\text { Vibrational couplings and energy transfer } \\
\text { pathways of water' s bending mode }\end{array}$ \\
\hline Author(s) & $\begin{array}{l}\text { Yu, Chun-Chieh; Chiang, Kuo-Yang; Okuno, } \\
\text { Masanari et al. }\end{array}$ \\
\hline Citation & Nature Communications. 11(1) p.5977 \\
\hline Issue Date & $2020-11-25$ \\
\hline oaire:version & VoR \\
\hline URL & https://hdl. handle.net/11094/78254 \\
\hline rights & $\begin{array}{l}\text { @ 2020 The Author(s). This article is Licensed } \\
\text { under a Creative Commons Attribution 4.0 } \\
\text { International License. }\end{array}$ \\
\hline Note & \multicolumn{1}{|l}{} \\
\hline
\end{tabular}

Osaka University Knowledge Archive : OUKA

https://ir. Library. osaka-u. ac. jp/

Osaka University 
ARTICLE

\title{
Vibrational couplings and energy transfer pathways of water's bending mode
}

\author{
Chun-Chieh Yu ${ }^{1,6}$, Kuo-Yang Chiang (1) 1,6, Masanari Okuno ${ }^{2 凶}$, Takakazu Seki (1) 1, Tatsuhiko Ohto ${ }^{3}$, \\ Xiaoqing $\mathrm{Yu}^{1}$, Vitaly Korepanov (10) ${ }^{4,5}$, Hiro-o Hamaguchi ${ }^{4}$, Mischa Bonn ${ }^{1}$, Johannes Hunger $\mathbb{1}^{1 凶} \&$ \\ Yuki Nagata (10 ${ }^{1 凶}$
}

Coupling between vibrational modes is essential for energy transfer and dissipation in condensed matter. For water, different $\mathrm{O}-\mathrm{H}$ stretch modes are known to be very strongly coupled both within and between water molecules, leading to ultrafast dissipation and delocalization of vibrational energy. In contrast, the information on the vibrational coupling of the $\mathrm{H}-\mathrm{O}-\mathrm{H}$ bending mode of water is lacking, even though the bending mode is an essential intermediate for the energy relaxation pathway from the stretch mode to the heat bath. By combining static and femtosecond infrared, Raman, and hyper-Raman spectroscopies for isotopically diluted water with ab initio molecular dynamics simulations, we find the vibrational coupling of the bending mode differs significantly from the stretch mode: the intramode intermolecular coupling of the bending mode is very weak, in stark contrast to the stretch mode. Our results elucidate the vibrational energy transfer pathways of water. Specifically, the librational motion is essential for the vibrational energy relaxation and orientational dynamics of $\mathrm{H}-\mathrm{O}-\mathrm{H}$ bending mode.

\footnotetext{
${ }^{1}$ Max Planck Institute for Polymer Research, Ackermannweg 10, 55128 Mainz, Germany. ${ }^{2}$ Department of Basic Science, Graduate School of Arts and Sciences, The University of Tokyo, 3-8-1 Komaba, Meguro, Tokyo 153-8902, Japan. ${ }^{3}$ Graduate School of Engineering Science, Osaka University, 1-3 Machikaneyama, Toyonaka, Osaka 560-8531, Japan. ${ }^{4}$ Department of Applied Chemistry and Institute of Molecular Science, National Chiao Tung University, 1001 Ta-Hsueh Road, Hsinchu 30010, Taiwan. ${ }^{5}$ Institute of Microelectronics Technology and High Purity Materials, RAS, Chernogolovka, Russia 142432.

${ }^{6}$ These authors contributed equally: Chun-Chieh Yu, Kuo-Yang Chiang. ${ }^{凶}$ email: cmokuno@mail.ecc.u-tokyo.ac.jp; hunger@mpip-mainz.mpg.de; nagata@mpip-mainz.mpg.de
} 
iquid water is important as a solvent, a solute, a reactant, and a catalyst, in the environment and many technological applications, but also in biology, driving protein folding and structuring nucleic acids. Many of the unique properties of water originate from the hydrogen bond (H-bond) network of water, which results from the strong intermolecular interactions between water molecules. For instance, the water H-bond network allows for rapid delocalization and dissipation of excess energy following chemical reactions, thereby making reactions irreversible. Insights into the flow of excess vibrational energy in water have therefore been deemed essential for understanding this anomalous liquid $^{1,2}$.

The vibrational modes of liquid $\mathrm{H}_{2} \mathrm{O}$ consist of the $\mathrm{O}-\mathrm{H}$ stretch mode, the $\mathrm{H}-\mathrm{O}-\mathrm{H}$ bending mode, the three librational modes, and collective modes below $100 \mathrm{~cm}^{-1}$. The $3000-4000 \mathrm{~cm}^{-1} \mathrm{O}-\mathrm{H}$ stretch mode has been most intensely studied, owing to its strong response ${ }^{3}$. Both experimental and simulation studies of the $\mathrm{O}-\mathrm{H}$ stretch mode have demonstrated different parallel pathways of vibrational energy transfer: within one water molecule, and between different water molecules ${ }^{1,4-7}$. Within one molecule, coupling can occur between the stretch modes of the two $\mathrm{O}-\mathrm{H}$ groups $^{8}$, and strong intermolecular coupling of stretch modes makes its excitation quasi-instantaneously delocalized across several molecules in liquid water. The stretch modes are further coupled to the bending mode and its overtone via anharmonic interactions ${ }^{9-17}$. As such, the bend vibration in liquid water is an important intermediate in the vibrational relaxation of the excited $\mathrm{O}-\mathrm{H}$ stretch vibration to the hot ground state ${ }^{18-21}$.

Remarkably, although the stretching mode has been intensely investigated, information on energy transfer pathways from the $\mathrm{H}-\mathrm{O}-\mathrm{H}$ bending mode is scarce. With approximately half the energy of the stretch mode, the bending mode likely provides an efficient pathway for energy delocalization and dissipation. The few experimental and theoretical studies of the bending mode reported to date have revealed that the delocalized character of $\mathrm{H}-\mathrm{O}-\mathrm{H}$ bending mode affects the vibrational dynamics ${ }^{16,22-24}$, but the impact of the delocalization on the dynamics is still under debate. For example, the impact of intermolecular bending-bending vibrational coupling (Fig. 1a) ${ }^{16,22,23,25}$ as well as vibrational coupling of the bending and librational modes (Fig. 1b, c) ${ }^{12-14,23,26}$ on energy relaxation have been separately investigated, while there is no systematic study for these mechanisms. Furthermore, the impact of the bending modelibrational mode mixing (Fig. 1d) on the dynamics has not been identified, though recent studies imply the importance of the mode mixing beyond the normal mode description ${ }^{15,16}$. This missing understanding of water's bending mode vibrational dynamics precludes a unified view of vibrational energy exchange and relaxation of water vibrations.

In this paper, we explore the energy dynamics of the bend vibration by directly comparing line broadening, vibrational energy relaxation dynamics, and randomization of the transition dipole orientation for the bending mode in $\mathrm{H}_{2} \mathrm{O}-\mathrm{D}_{2} \mathrm{O}$ mixtures. Lowering the $\mathrm{H}_{2} \mathrm{O}$ concentration allows suppressing the effect of intermolecular coupling, while keeping the local environment nearly unchanged. Our results show that the intermolecular coupling of the bend vibration is relatively weak in contrast to the stretch vibration. In line with earlier reports ${ }^{1,3,27}$, we find intermolecular vibrational energy relaxation to occur on a (sub-) picosecond timescale. Yet, our results suggest that intermolecular bend-to-bend energy transfer is much slower ( $\sim 1 \mathrm{ps})$ than the intermolecular stretch-to-stretch transfer ( $0.1 \mathrm{ps})$. Randomization of the orientations for the $\mathrm{H}-\mathrm{O}-\mathrm{H}$ bending mode transition dipole moment occurs much faster, which is attributed to rotational contributions (e.g., librational mode) to the bending mode band.

\section{Results}

Variation of FTIR, Raman, and hyper-Raman spectra of water bending mode due to vibrational couplings. The linewidth of a vibrational band is the most obvious reporter of vibrational couplings. By varying the $\mathrm{H} / \mathrm{D}$ isotopic ratio in water, one can modulate the vibrational couplings of water molecules systematically, while keeping the local environment virtually unaffected $^{6}$. Using this approach, the delocalization of the $\mathrm{O}-\mathrm{H}$ stretch mode $7,10,28,29$ has been demonstrated. This delocalization is also responsible for a significant broadening of the $\mathrm{O}-\mathrm{H}$ stretch a

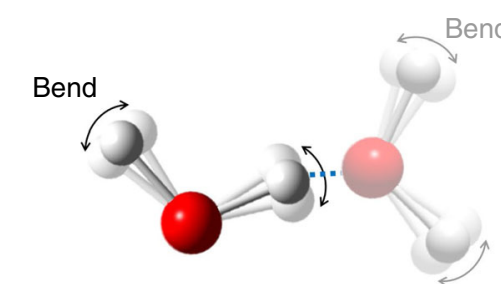

Intermolecular bend-bend coupling

C

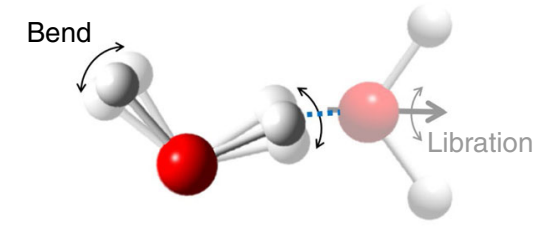

Intermolecular bend-libration coupling b

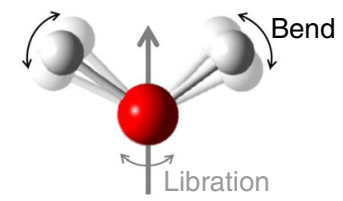

Intramolecular bend-libration coupling

d

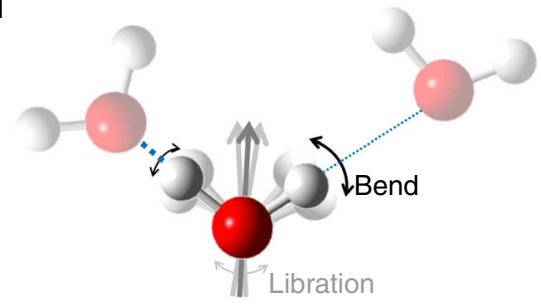

Bend-libration mixing through asymmetric $\mathrm{H}$-bond

Fig. 1 Types of the vibrational couplings of the $\mathbf{H}-\mathbf{O}-\mathbf{H}$ bending mode. a Intermolecular bend-bend coupling of different water molecules. $\mathbf{b}$ Intramolecular bend-libration coupling within a single water molecule. $\mathbf{c}$ Intermolecular bend-libration coupling of different water molecules. $\mathbf{d}$ Bend-libration mixing induced by the asymmetric hydrogen-bond ( $\mathrm{H}$-bond) strengths. Hydrogen and Oxygen atoms are represented by white and red spheres, respectively. The gray arrows represent the $\mathrm{H}-\mathrm{O}-\mathrm{H}$ angular bisector direction. Strong (weak) hydrogen-bonds are represented by thick (thin) broken blue lines. 

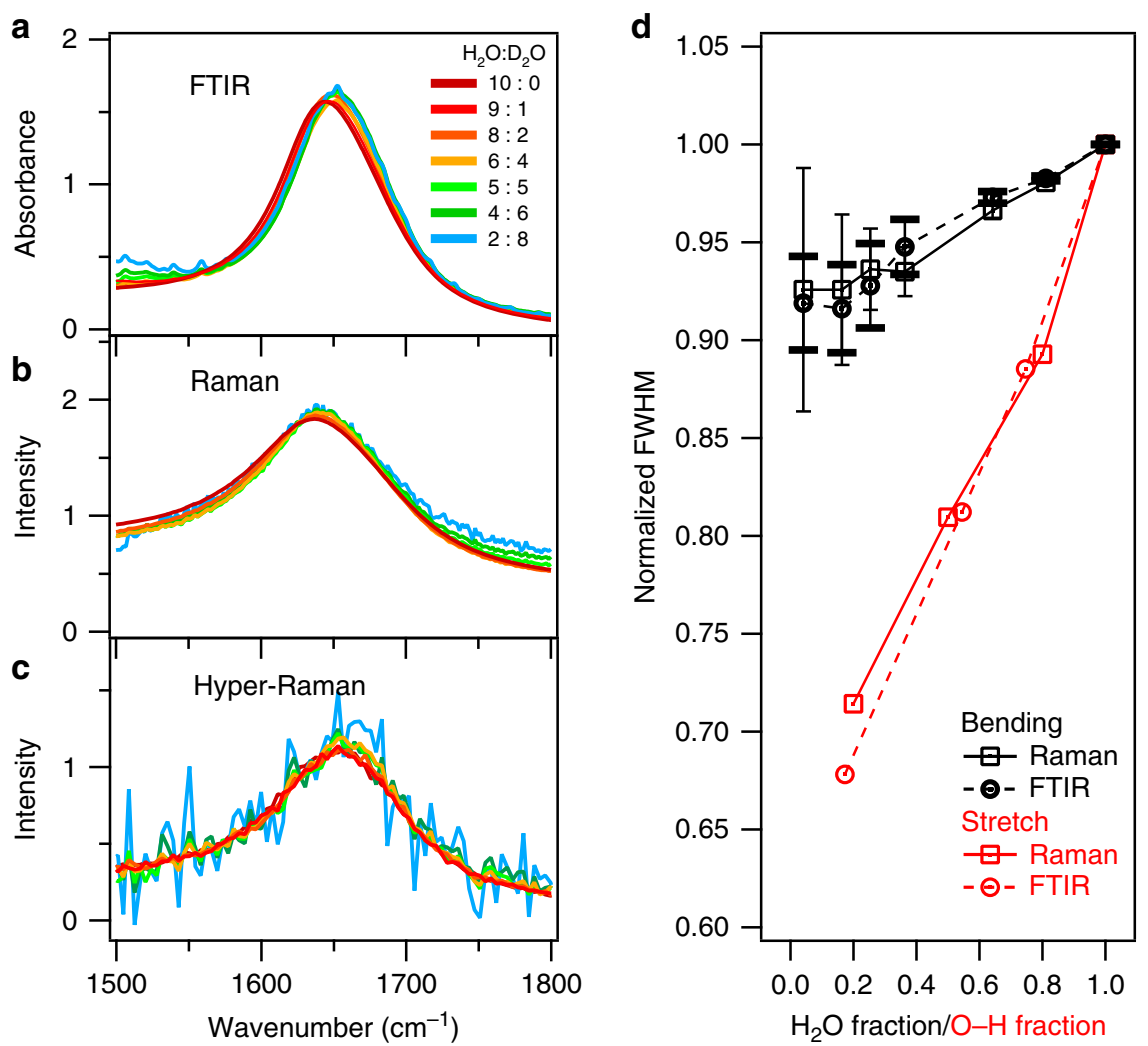

Fig. 2 The extracted $\mathbf{H}-\mathbf{O}-\mathbf{H}$ bending mode contributions. a IR, $\mathbf{b}$ Raman, and $\mathbf{c}$ hyper-Raman spectra of various $\mathrm{H}_{2} \mathrm{O}-\mathrm{D}_{2} \mathrm{O}$ mixtures. $\mathbf{d}$ Comparison of the full widths at half maximum (FWHM) for the $\mathrm{H}-\mathrm{O}-\mathrm{H}$ bending mode contributions and the $\mathrm{O}-\mathrm{H}$ stretch mode spectra. The O-H stretch IR and Raman data are extracted from refs. ${ }^{49,50}$, respectively. The error bars correspond to the uncertainty of the FWHMs when using the sample with the different concentration for obtaining the $\mathrm{H}-\mathrm{O}-\mathrm{D}$ bending spectrum for the background subtraction (see Supplementary Note 7).

band for pure $\mathrm{H}_{2} \mathrm{O}$. Using isotopic dilution, the vibrational coupling of the bending mode of water can also be modulated. The contributions of background-corrected $\mathrm{H}-\mathrm{O}-\mathrm{H}$ bending mode in FTIR, Raman, and hyper-Raman spectra of $\mathrm{H}_{2} \mathrm{O}-\mathrm{D}_{2} \mathrm{O}$ mixtures are shown in Fig. $2 \mathrm{a}-\mathrm{c}$, respectively. The methodology to subtract the background as well as the HDO and $\mathrm{D}_{2} \mathrm{O}$ contributions from the measured spectra is detailed in the Supplementary Note 6. The IR, Raman, and hyper-Raman responses for the $\mathrm{H}-\mathrm{O}-\mathrm{H}$ bending mode in neat $\mathrm{H}_{2} \mathrm{O}$ peak at 1644,1637 , and $1656 \mathrm{~cm}^{-1}$, respectively. The variation in the peak frequency between the IR, Raman, and hyper-Raman spectra reflects the different frequency-dependence of the transition dipole moment, polarizability, and hyper-polarizability - the so-called non-coincidence effect - and also signifies coupling ${ }^{30,31}$. While the peak frequencies are different for the three techniques, the blue-shift of the resonance upon isotopic dilution is very similar. The similarity suggests that the underlying vibrational couplings in water have a common origin ${ }^{32,33}$. The insensitivity of the magnitude of the shift to the experimental details is consistent with the notion that intermolecular bend-bend coupling is not of dipolar nature but has mechanic or electronic origins ${ }^{32}$. Accordingly, the same blue-shift can be observed in the aqueous salt solutions (see Supplementary Figs. 10 and 11).

The variations of the full widths at half maximum (FWHM) are summarized in Fig. 2d. The FWHMs of the $\mathrm{H}-\mathrm{O}-\mathrm{H}$ bending mode decrease in both the IR and Raman spectra upon the addition of $\mathrm{D}_{2} \mathrm{O}$. This means that the vibrational coupling of the bending mode not only shifts the peak frequency but also broadens the linewidths. On the other hand, the variation of the FWHM for the bending mode is much smaller than that of the $\mathrm{O}-\mathrm{H}$ stretch mode which narrows dramatically upon addition of
$\mathrm{D}_{2} \mathrm{O}$. The small FWHM variation of the bending mode, compared to the stretch mode, can be attributed to weaker intermolecular intramode transition dipole interaction resulting from the smaller transition dipole moment for the bending mode, relative to the $\mathrm{O}-\mathrm{H}$ stretch mode ${ }^{34}$. Furthermore, the degree of narrowing is much smaller than expected from previous theoretical predictions ${ }^{32}$. These observations show that the vibrational coupling effects on the peak width and position are modest, yet clearly present in the static spectra.

Energy relaxation from water bending mode to lower frequency mode determined by pump-probe IR measurement. To disentangle these vibrational couplings of the $\mathrm{H}-\mathrm{O}-\mathrm{H}$ bending mode, we carried out time-resolved IR measurement by pumping and probing the $\mathrm{H}-\mathrm{O}-\mathrm{H}$ bending mode in neat $\mathrm{H}_{2} \mathrm{O}$ and isotopically diluted water. In these experiments, an intense pump laser pulse excites the bending mode from the vibrational ground state to the first vibrationally excited state, and transient spectral modulations are probed as a function of delay time, $t$. The excitation reduces absorption at the fundamental frequency of the bending mode (ground state bleach) and increases absorption (excited state absorption) at red-shifted frequencies. We measured the transient absorption spectra with the polarization of the probe light both perpendicular $\left(\Delta \alpha_{\perp}(\omega, t)\right)$ and parallel $\left(\Delta \alpha_{\|}(\omega, t)\right)$ directions to that of the excitation pulse.

First, we discuss the vibrational energy relaxation (intramode energy transfer) of the $\mathrm{H}-\mathrm{O}-\mathrm{H}$ bending mode. In neat $\mathrm{H}_{2} \mathrm{O}$, the vibrational energy transfer from the bending mode to librational mode occurs within a single water molecule (intramolecularly, Fig. 1b) and through energy relaxation from the bending mode of 

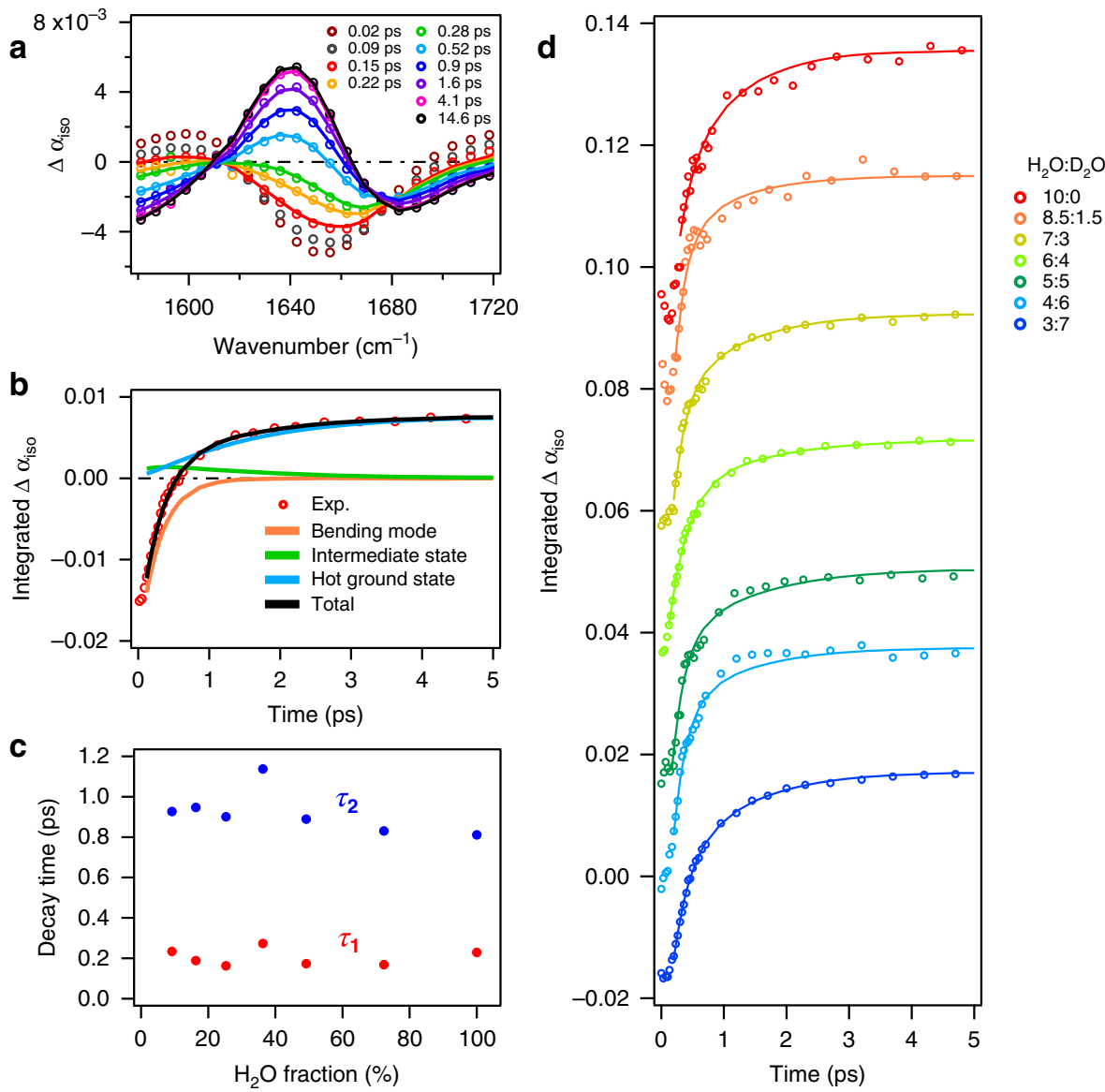

Fig. 3 The isotropic transient absorption of various isotopic compositions. a Transient absorption spectra of water bending mode for $\mathrm{H}_{2} \mathrm{O}: \mathrm{D}_{2} \mathrm{O}=6: 4$. The four-state model fit the data starting at $0.15 \mathrm{ps}$. b Time evolution of the transient absorption spectra integrated at $1649 \mathrm{~cm}^{-1}<\omega<1662 \mathrm{~cm}^{-1}$. The orange, green, and blue lines represent the contributions of the excited state, intermediate state, and hot ground state, respectively, to the fit. $\mathbf{c}$ Isotropic relaxation times as a function of isotopic composition. The relaxation time of the excited state to intermediate state is $\tau_{1}$ (red symbol) and the relaxation time of the intermediate state is $\tau_{2}$ (blue symbol). $\mathbf{d}$ Isotropic transient signals for various $\mathrm{H}_{2} \mathrm{O}-\mathrm{D}_{2} \mathrm{O}$ mixtures. The signals are normalized at $t=0$. Traces are offset by increments of 0.02 . Open circles in panels $\mathbf{a}, \mathbf{b}$ and $\mathbf{d}$ represent the experimental data, while the solid lines show fits with the kinetic model.

one water molecule to the librational mode of an adjacent water molecule (intermolecularly, Fig. 1c) ${ }^{12-14,23,26}$. Through isotopic dilution of water, the intermolecular intermode transfer from the bending mode of the $\mathrm{H}_{2} \mathrm{O}$ molecule to the librational mode of the surrounding water molecules is altered, because the isotopic dilution shifts the librational mode frequency of $\mathrm{H}_{2} \mathrm{O}$ to that of $\mathrm{D}_{2} \mathrm{O}$ and $\mathrm{HDO}$ (see also Supplementary Figs. 2 and 3), changing the vibrational coupling between the bending mode and librational mode.

Figure 3a shows the isotropic component of the transient absorption response, $\Delta \alpha_{\text {iso }}(\omega, t)=\left[\Delta \alpha_{\|}(\omega, t)+2 \Delta \alpha_{\perp}(\omega, t)\right] / 3$, which solely represents energy relaxation and is free from rotational contributions, of the $\mathrm{H}-\mathrm{O}-\mathrm{H}$ bending mode for the $\mathrm{H}_{2} \mathrm{O}: \mathrm{D}_{2} \mathrm{O}=6: 4$ sample. With increasing delay time, the excited state population relaxes to the ground state, and the dissipated energy populates low frequency modes, which results in persistent spectral modulations at long delay times. To quantify the vibrational dynamics, we used a four-state kinetic model similar to previous studies ${ }^{12,14}$, in which the four states represent, respectively, ground state, bending mode excited state, intermediate state (e.g. including the librational mode excited state), and hot ground state. Figure $3 \mathrm{~b}$ shows integrated $\left(1649 \mathrm{~cm}^{-1}<\right.$ $\left.\omega<1662 \mathrm{~cm}^{-1}\right) \Delta \alpha_{\text {iso }}$ signals as a function of delay time together with the overall fit and the contributions of each state of the model (for details on the fit model, see Supplementary Note 8); the fit manifests that the four-level model describes the experimental data very well (Fig. 3a, b). Figure 3d displays the integrated $\Delta \alpha_{\text {iso }}(\omega, t)$ for various concentrations of $\mathrm{H}_{2} \mathrm{O}-\mathrm{D}_{2} \mathrm{O}$ mixtures. The time traces are similar for all samples. The relaxation time from the excited state bending mode to the intermediate state $\left(\tau_{1}\right)$ is $\sim 200 \mathrm{fs}$ (Fig. 3c), consistent with previous reports ${ }^{14,16}$. Within error, $\tau_{1}$ is insensitive to the isotopic composition. The insensitivity of $\tau_{1}$ to $\mathrm{H}_{2} \mathrm{O}$ concentration reveals that the energy relaxation process is unaffected by the isotopic composition of the water molecules surrounding the excited chromophore. This suggests that intramolecular (Fig. 1b), but not intermolecular (Fig. 1c), bend-libration coupling dictates the vibrational energy relaxation of the bending mode.

Intermolecular bend-to-bend energy transfer measured by polarization-dependent pump-probe IR measurement. Figure 4 a shows the time traces of the anisotropy decay for different isotopic substitutions. The decay of the excitation anisotropy of the $\mathrm{H}-\mathrm{O}-\mathrm{H}$ bending mode, i.e., the orientational memory of the anisotropic excitation due to polarized light, has provided detailed insight into the coupling of the $\mathrm{H}-\mathrm{O}-\mathrm{H}$ bending chromophores 7,35 . This anisotropy decay is defined as;

$$
R(t)=\int_{\omega_{1}}^{\omega_{2}} \frac{\Delta \alpha_{\|}^{\prime}(\omega, t)-\Delta \alpha_{\perp}^{\prime}(\omega, t)}{\Delta \alpha_{\|}^{\prime}(\omega, t)+2 \Delta \alpha_{\perp}^{\prime}(\omega, t)} \mathrm{d} \omega
$$


a

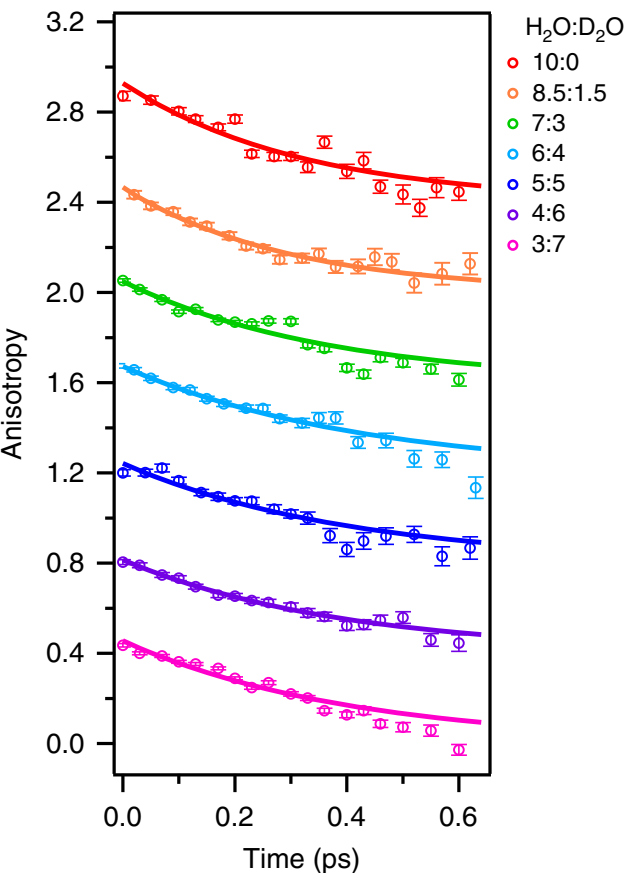

b

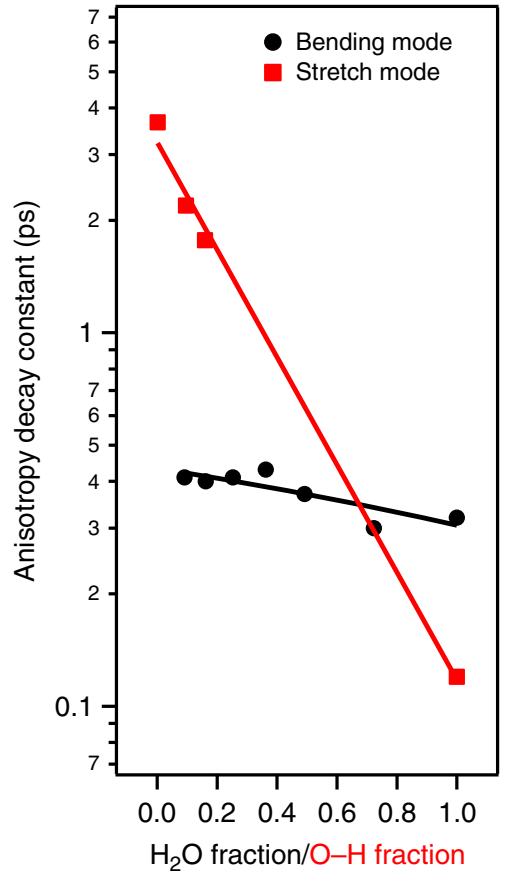

c

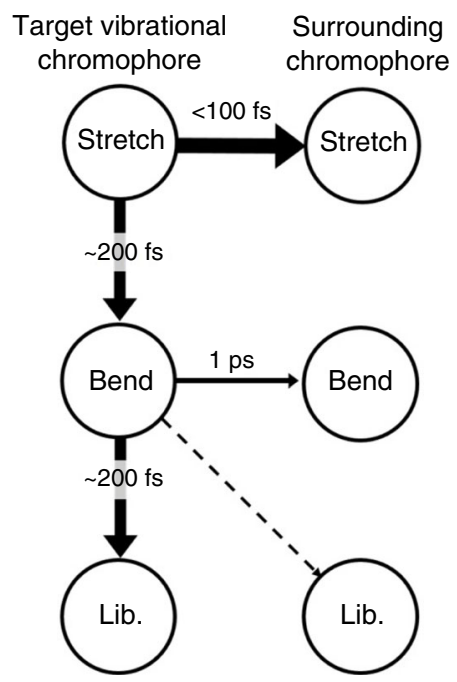

Fig. 4 The anisotropy decay of various isotopic composition and schematic for the vibrational energy relaxation of water molecules. a Anisotropy decay for various $\mathrm{H}_{2} \mathrm{O}-\mathrm{D}_{2} \mathrm{O}$ mixtures. To obtain the excitation anisotropy, the signals arising from the hot ground state were subtracted from the raw data. Traces are offset by increments of 0.4. Symbols show experimental data and solid lines show fits using a single exponential decay. $\mathbf{b}$ Comparison of the anisotropy decay time for the bending mode and the stretch mode as a function of $\mathrm{H}_{2} \mathrm{O} / \mathrm{O}-\mathrm{H}$ fraction. The $\mathrm{H}_{2} \mathrm{O} / \mathrm{O}-\mathrm{H}$ fraction represent the mole fraction of $\mathrm{H}_{2} \mathrm{O}$ molecules and of $\mathrm{O}-\mathrm{H}$ stretch groups for various $\mathrm{H}_{2} \mathrm{O}-\mathrm{D}_{2} \mathrm{O}$ mixtures, respectively. The stretch mode decay times were obtained by fitting an exponential decay to the data reported in ref. ${ }^{7}$. The lines serve to guide the eye. c Schematic representation for the vibrational energy relaxation of $\mathrm{H}-\mathrm{O}-\mathrm{H}$ molecules in neat water. The stretch mode timescales were taken from refs. ${ }^{15,27,51,52}$. The target vibrational chromophore is the initial excited $\mathrm{H}-\mathrm{O}-\mathrm{H}$ molecule. The surrounding chromophores are the water molecules surrounding the excited molecule.

where $\Delta \alpha_{\perp}^{\prime}(\omega, t)$ and $\Delta \alpha_{\|}^{\prime}(\omega, t)$ represent the transient spectra after the effects of the spectral contribution of the hot ground state (i.e., the spectral signature at long $t$, in Fig. $3 \mathrm{~b}$ ) were subtracted from $\Delta \alpha_{\perp}(\omega, t)$ and $\Delta \alpha_{\|}(\omega, t)$, respectively. We set $\omega_{1}=1649 \mathrm{~cm}^{-1}$ and $\omega_{2}=1662 \mathrm{~cm}^{-1}$ in Eq. (1), similar to a previous study ${ }^{16}$.

In contrast to the isotropic transient signals, the excitation anisotropy provides insight into the randomization of the transition dipole orientation of the excited chromophore. Such orientational randomization for neat $\mathrm{H}_{2} \mathrm{O}$ can arise from three mechanisms; firstly, the rotational motion of water, secondly the loss of the orientational information through exciton hopping to a neighboring water molecule with a differently aligned transition dipole (facilitated by intermolecular bend-to-bend coupling, Fig. 1a), and thirdly the orientational fluctuations caused by bend-libration mixing (Fig. 1d), which all result in a decay of the excitation anisotropy. In isotopically diluted water, the contribution due to intermolecular bend-to-bend energy transfer is suppressed, because of the frequency mismatch of the $\mathrm{H}-\mathrm{O}-\mathrm{H}$ bending mode and $\mathrm{H}-\mathrm{O}-\mathrm{D} / \mathrm{D}-\mathrm{O}-\mathrm{D}$ bending modes. As such, in analogy to the $\mathrm{O}-\mathrm{H}$ stretching band $\mathrm{d}^{7,35}$, one can quantify the intermolecular bend-to-bend energy transfer from the anisotropy decay of different isotopic composition.

The time evolution of the anisotropy is displayed in Fig. 4a. The anisotropy of $\mathrm{H}_{2} \mathrm{O}$ molecules in neat $\mathrm{H}_{2} \mathrm{O}$ decays with a timescale of $\sim 300 \mathrm{fs}$. This decay is somewhat slower than that previously reported for neat $\mathrm{H}_{2} \mathrm{O}^{16,22}$, which can likely be attributed to the different procedure to subtract the contributions of the hot ground state from the data (see the Supplementary Notes 8 and 9). Consistent with these reports, and despite the short vibrational relaxation time limiting the accessible time window for determining the excitation anisotropy to $t<600 \mathrm{fs}$, we find the anisotropy to decay faster than what would be expected based on rotation of water (i.e., the $\mathrm{H}-\mathrm{O}-\mathrm{H}$ angular bisector orientational decay of $1.9 \mathrm{ps}^{36}$ ). The anisotropy decay times, as obtained from a fit of an exponential decay to the data, slows down from $310 \pm 20$ fs to $440 \pm 20$ fs upon addition of $\mathrm{D}_{2} \mathrm{O}$. This is in stark contrast with the $\mathrm{O}-\mathrm{H}$ stretch mode, which shows $>10$ times faster anisotropy decay in neat $\mathrm{H}_{2} \mathrm{O}$ than in isotopically diluted water due to the stretch-stretch modes coupling/energy transfer (see Fig. 4b) ${ }^{1,7}$. The weaker variation of the anisotropy with isotopic composition may be rationalized by the smaller transition dipole moment of the bending mode of water. Assuming that the variation of the anisotropy decay can be fully ascribed to intermolecular bend-to-bend energy transfer, we estimated the time constant of bend-to-bend energy transfer in neat water from the difference in the fitted decay rates at $\mathrm{H}_{2} \mathrm{O}$ fractions of 0 and 1 . This provides the time constant of $\left((310 \mathrm{fs})^{-1}-(440 \mathrm{fs})^{-1}\right)^{-1}=1.05 \pm 0.26 \mathrm{ps}$. As such, the intermolecular intramode transfer is rather slow, in stark contrast to the $\mathrm{O}-\mathrm{H}$ stretch mode, exhibiting strong intermolecular intramode vibrational couplings. In turn, the vibrational energy transfer pathway from $\mathrm{H}_{2} \mathrm{O}$ bending modes seems to be governed by the energy relaxation to the librational motion. This is summarized in Fig. 4c.

\section{Discussion}

The fact that the anisotropy of the bending excitation decays on a 440 -fs time scale, which is much faster than the reorientation time of a water molecule $\left(\sim 1.9 \mathrm{ps}^{36}\right)$ strongly suggests that 

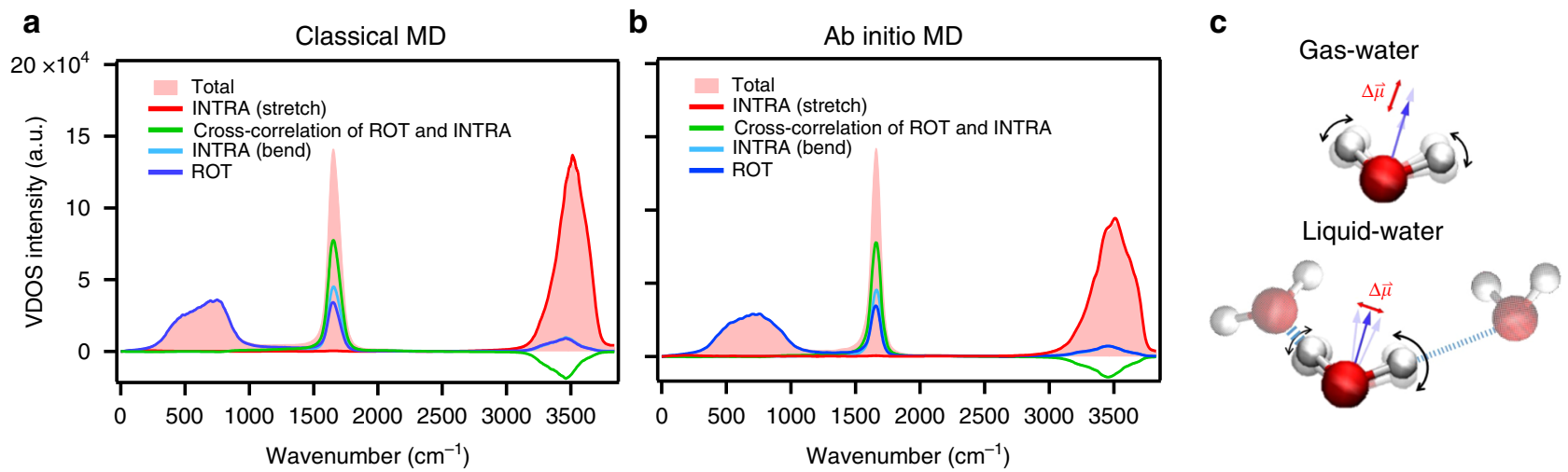

Fig. 5 Decomposed vibrational density of states (VDOS) spectra of neat $\mathbf{H}_{\mathbf{2}} \mathbf{O}$. a Classical force field MD with the POLI2VS model 53 and $\mathbf{b} A I M D$ simulation at the revPBEO-D3(0) level of theory. The frequency of the POLI2VS model is scaled with a factor of 0.96 to account for nuclear quantum effects. The frequencies of the bending and stretch modes for the revPBEO-D3(0) model are corrected via the procedure described in ref. ${ }^{54}$. The red region illustrates the total VDOS spectrum of neat $\mathrm{H}_{2} \mathrm{O}$. The differently colored lines represent the contribution of different motions. c Schematics of water molecules in the gas and liquid phase. Strong (weak) hydrogen-bonds are represented by thick (thin) broken lines. The blue arrows represent the direction of the dipole moment of the highlighted water molecule, while the red lines represent the direction of the change in the dipole moment (transition dipole moment, $\Delta \vec{\mu}$ ).

vibrational modes other than the pure bending motion contribute to the observed band at $\sim 1650 \mathrm{~cm}^{-1} 16$. If the $1650 \mathrm{~cm}^{-1}$ band contains vibrational contributions other than a pure bending motion of water's protons, the detected anisotropy decay does not solely arise from the pure rotational motion of the water molecule, rationalizing the anisotropy decay of the bending mode to be faster than the rotation times as obtained from, e.g., IR pumpprobe experiments on isotopically diluted HOD molecules and NMR experiments ${ }^{37,38}$.

To elucidate the exact origin of the vibrational response at $1500-1600 \mathrm{~cm}^{-1}$, we computed the vibrational density of states (VDOS) spectra from the velocity autocorrelation as obtained from ab initio molecular dynamics (AIMD) simulations at the revPBE0-D3(0) level of theory as well as from classical force field simulations with the POLI2VS model. We decomposed the vibrational response based on the decomposition of the velocity into the components that arise from the rotational motion (ROT) of an entire water molecule and the intramolecular motion (INTRA). We further decomposed the INTRA motion into the $\mathrm{O}-\mathrm{H}$ stretching motion that goes along with a variation of the $\mathrm{O}-\mathrm{H}$ distances and into bending motion contributions, which mainly involves the variation of the $\mathrm{H}-\mathrm{H}$ distance. The subsequent decomposed VDOS spectra are displayed in Fig. 5a, b (for computational details, see Supplementary Note 5).

The simulated VDOS spectra show that the $1650 \mathrm{~cm}^{-1}$ feature is not only governed by the intramolecular vibrational motion but also contains contributions from the rotational motion of water molecules. Interestingly and perhaps surprisingly, the pure bending motion, which is not accompanied by a simultaneous rotational motion of water, only accounts for $30 \%$ of the $1650 \mathrm{~cm}^{-1}$ VDOS feature. The largest contribution arises from the cross-correlation terms of the bending motion and rotational motion through the mixing of the bending mode and librational mode (see Fig. 1d). As such, the simulations confirm the strong coupling of the bending mode to water's librations, giving rise to the $1650 \mathrm{~cm}^{-1}$ band. The marked rotational contributions to the $1650 \mathrm{~cm}^{-1}$ peak can be rationalized by the notion that - in contrast to water in the gas phase - in liquid water the heterogeneity of hydrogen-bond strengths around a water molecule makes the bending mode potential asymmetric with respect to the bisector (Fig. $5 \mathrm{c})^{39,40}$. The vibrational coordinate of the $1650 \mathrm{~cm}^{-1}$ mode contains both rotational motions of the water molecule and a modulation of the $\mathrm{H}-\mathrm{H}$ distance. In contrast to the band at $1650 \mathrm{~cm}^{-1}$, the band at
$3200-3600 \mathrm{~cm}^{-1}$ can be primarily explained by a proton motion along the $\mathrm{O}-\mathrm{H}$ axis, that is, the stretching vibration (Fig. 5a, b).

Together, the rotational contributions to the $1650 \mathrm{~cm}^{-1}$ mode make the orientation of its transition dipole to differ from the $\mathrm{H}-\mathrm{O}-\mathrm{H}$ angular bisector (Fig. 5c), and the anisotropy decay of the pump-probe experiments probes the motion of an axis different from the $\mathrm{H}-\mathrm{O}-\mathrm{H}$ angular bisector directions. Since the orientation of the transition dipole moment varies with the hydrogen bond strength of water as schematically depicted in the bottom panel of Fig. $5 c$, the acceleration of the anisotropy decay with respect to the pure $\mathrm{H}-\mathrm{O}-\mathrm{H}$ bisector orientational motion can be linked to the hydrogen bond dynamics. In fact, the acceleration can be estimated to $\left((440 \mathrm{fs})^{-1}-(1.9 \mathrm{ps})^{-1}\right)^{-1}=570 \mathrm{fs}$. The 570 -fs time scale agrees with the time constant of the hydrogen bond dynamics of $0.5-1.0 \mathrm{ps}$, as characterized by the spectral diffusion for the $\mathrm{O}-\mathrm{H}$ stretch of dilute $\mathrm{HOD}$ in $\mathrm{D}_{2} \mathrm{O}^{28,41-43}$. As such, large bending-libration mode mixing rationalizes the observation that the excitation anisotropy for the $1650 \mathrm{~cm}^{-1}$ band ( $440 \mathrm{fs}$ ) of isotopically diluted water as measured in the pump-probe IR measurement decays much faster than the orientation of the bisector as measured using NMR $(\sim 1.9 \mathrm{ps})^{36}$. This large bending-librational mode mixing causes the $10 \%$ line broadening of the static bending mode spectra (see Fig. 2). In contrast, the weak mode mixing for the stretch mode of isotopically diluted water is consistent with the observation that the anisotropy decay for the of the $\mathrm{O}-\mathrm{H}$ stretch mode at $\sim 3400 \mathrm{~cm}^{-1}$ for isotopically diluted water $\left(\approx 3-4 \mathrm{ps}^{7,41,44}\right)$ is comparable to the orientational correlation time as detected with NMR (decay $\approx 2 \mathrm{ps}^{45-48}$ ).

To summarize, we quantified the vibrational coupling of the water bending mode employing static and time-resolved vibrational spectroscopic techniques. The IR, Raman, and hyper-Raman measurements of water bending mode show that the vibrational coupling of the bending mode blue-shifts the peak position by $\sim 8 \mathrm{~cm}^{-1}$ and reduces the linewidth by $10 \%$, upon isotopic dilution. The timeresolved IR measurements reveal that the intermolecular bend-tobend (intramode) coupling is much weaker than the intermolecular stretch-to-stretch coupling. The intramolecular bend-to-libration energy transfer $(\approx 200 \mathrm{fs})$ takes place much faster than the intermolecular bend-to-bend coupling ( $\approx 1 \mathrm{ps})$. As such, the vibrational energy of bending mode is mainly released not to the bending modes of surrounding water molecules but to the librational mode, in stark contrast to the stretch mode. AIMD simulations confirm strong bend-libration coupling, which can explain the faster decay of the excitation anisotropy of the bending mode $(\approx 0.4 \mathrm{ps})$, much 
faster than for the stretch mode $(\approx 2-4 \mathrm{ps})$. The experimental and simulation results demonstrate that the librational mode plays a key role in the energy transfer pathway and orientational dynamic of the bending mode band.

\section{Methods}

FTIR, Raman, and hyper-Raman experiment. For the FTIR experiment, FTIR spectra were recorded with a Bruker VERTEX 70 FTIR spectrometer in transmission. The samples were held between two $\mathrm{CaF}_{2}$ windows separated by a $15-\mu \mathrm{m}$ thick Teflon spacer. The spectrometer was purged with $\mathrm{N}_{2}$ and spectra were recorded at $298 \mathrm{~K}$. For the Raman experiment, the excitation was made with a 532-nm laser (Millennia eV, Spectra-Physics). The signal was dispersed in a spectrometer (SR303i-B, Andor) and detected by a CCD camera (DU420A-BVF, Andor). We measured the spectra in the parallel polarization configuration. For the hyper-Raman experiment, we used a picosecond laser (Cepheus 1002, Photon Energy, $1064 \mathrm{~nm}, \sim 15 \mathrm{ps}, 150 \mathrm{kHz})$. The output was frequency-doubled $(532 \mathrm{~nm})$ and then focused into the sample. The signal was dispersed in a spectrometer (iHR320, Horiba) and detected by a CCD camera (DU420A-BVF, Andor). The details can be found in the Supplementary Notes 1-3.

Pump-probe IR experiment. The measurements were performed on a femtosecond Ti: Sapphire amplified laser system (Coherent Astrella, $\sim 800 \mathrm{~nm}, \sim 35 \mathrm{fs}$, $1 \mathrm{kHz}$ ) with $6.8 \mathrm{~W}$ output power. In all, $2.8 \mathrm{~W}$ of the output was used to pump an optical parametric amplifier (TOPAS, light conversion) with a non-collinear DFG stage to generate broadband IR pulses (centered at $1600 \mathrm{~cm}^{-1}, 100 \mathrm{fs}$ duration, $17 \mu \mathrm{J}$ pulse energy, and $300 \mathrm{~cm}^{-1}$ full width at half maximum (FWHM)). The IR pulses were split into probe $(\sim 2.5 \%)$, reference $(\sim 2.5 \%)$, and pump pulses $(\sim 95 \%)$. The polarization of the pump-pulse was set at $45^{\circ}$ with respect to the probe pulse polarization using a half-wave plate. The sample was placed between two $\mathrm{CaF}_{2}$ windows separated by a $15-\mu \mathrm{m}$-thick Teflon spacer. A wire grid polarizer mounted in a rotating stage allowed us to select the parallel and perpendicular polarization components of the probe beam, relative to the pump polarization. Both the probe and the reference pulses were dispersed with a spectrometer onto a liquid-nitrogencooled mercury-cadmium telluride detector. The details can be found in the Supplementary Note 4.

MD Simulation. We used the AIMD trajectories at the revPBE0-D3(0) level of theory as well as the classical force field molecular dynamics trajectories with the POLI2VS model. The simulation cell contained $64 \mathrm{H}_{2} \mathrm{O}$ in the AIMD, while the POLI2VS simulation cell contained $500 \mathrm{H}_{2} \mathrm{O}$ molecules in the POLI2VS simulation. The target temperature was set to $300 \mathrm{~K}$. The VDOS spectra were calculated for the dipole moment directions via;

$$
\begin{gathered}
\operatorname{VDOS}(\omega)=\int_{0}^{T} \cos (\omega t) \cos ^{2}\left(\frac{\pi t}{2 T}\right)\left\langle\Sigma_{i} \mathbf{v}_{i}(t) \cdot \mathbf{v}_{i}(0)\right\rangle \mathrm{d} t, \\
\mathbf{v}_{i}(t)=\frac{\left(\mathbf{v}_{i, \mathrm{H} 1}(t)+\mathbf{v}_{i, \mathrm{H} 2}(t)\right)}{2}-\mathbf{v}_{i, \mathrm{O}}(t),
\end{gathered}
$$

where $\mathbf{v}_{i, x}(t)$ denotes the velocity vector of atom $x=\mathrm{O}, \mathrm{H}_{1}, \mathrm{H}_{2}$ for water molecule $i$. The VDOS spectra were decomposed based on the center of mass velocity $\mathbf{v}_{i, \mathrm{COM}}(t)$, rotational motion velocity $\mathbf{v}_{i, \mathrm{ROT}}(t)$, intramolecular bending motion velocity $\mathbf{v}_{i, \text { INTRA(bend) }}(t)$, and intramolecular stretch motion velocity $\mathbf{v}_{i, \text { INTRA (stretch) }}(t)$. The details can be found in the Supplementary Note 5.

\section{Data availability}

The data that support the findings of this study are available from the corresponding authors upon reasonable request.

Received: 16 July 2020; Accepted: 26 October 2020;

Published online: 25 November 2020

\section{References}

1. Perakis, F. et al. Vibrational spectroscopy and dynamics of water. Chem. Rev. 116, 7590-7607 (2016)

2. Nihonyanagi, S., Yamaguchi, S. \& Tahara, T. Ultrafast dynamics at water interfaces studied by vibrational sum frequency generation spectroscopy. Chem. Rev. 117, 10665-10693 (2017).

3. Rey, R., Møller, K. B. \& Hynes, J. T. Hydrogen bond dynamics in water and ultrafast infrared spectroscopy: a theoretical study. J. Phys. Chem. A 106, 11993-11996 (2002).

4. Auer, B. M. \& Skinner, J. L. IR and Raman spectra of liquid water: theory and interpretation. J. Chem. Phys. 128, 224511 (2008).
5. Bakker, H. J. \& Skinner, J. L. Vibrational spectroscopy as a probe of structure and dynamics in liquid water. Chem. Rev. 110, 1498-1517 (2010).

6. Matt, S. M. \& Ben-Amotz, D. Influence of intermolecular coupling on the vibrational spectrum of water. J. Phys. Chem. B 122, 5375-5380 (2018).

7. Woutersen, S. \& Bakker, H. J. Resonant intermolecular transfer of vibrational energy in liquid water. Nature 402, 507-509 (1999).

8. DeMarco, L. et al. Differences in the vibrational dynamics of $\mathrm{H}_{2} \mathrm{O}$ and $\mathrm{D}_{2} \mathrm{O}$ observation of symmetric and antisymmetric stretching vibrations in heavy water. J. Phys. Chem. Lett. 7, 1769-1774 (2016).

9. Kananenka, A. A. \& Skinner, J. L. Fermi resonance in $\mathrm{OH}$-stretch vibrational spectroscopy of liquid water and the water hexamer. J. Chem. Phys. 148, 244107 (2018)

10. Schaefer, J., Backus, E. H. G., Nagata, Y. \& Bonn, M. Both inter- and intramolecular coupling of $\mathrm{O}-\mathrm{H}$ groups determine the vibrational response of the water/air interface. J. Phys. Chem. Lett. 7, 4591-4595 (2016).

11. Ahmed, M., Singh, A. K., Mondal, J. A. \& Sarkar, S. K. Water in the hydration shell of halide ions has significantly reduced fermi resonance and moderately enhanced Raman cross section in the $\mathrm{OH}$ stretch regions. J. Phys. Chem. B 117, 9728-9733 (2013).

12. Ashihara, S., Huse, N., Espagne, A., Nibbering, E. T. J. \& Elsaesser, T. Vibrational couplings and ultrafast relaxation of the $\mathrm{O}-\mathrm{H}$ bending mode in liquid $\mathrm{H}_{2} \mathrm{O}$. Chem. Phys. Lett. 424, 66-70 (2006).

13. Ashihara, S., Fujioka, S. \& Shibuya, K. Temperature dependence of vibrational relaxation of the $\mathrm{OH}$ bending excitation in liquid $\mathrm{H}_{2} \mathrm{O}$. Chem. Phys. Lett. 502, 57-62 (2011).

14. Huse, N., Ashihara, S., Nibbering, E. T. J. \& Elsaesser, T. Ultrafast vibrational relaxation of $\mathrm{O}-\mathrm{H}$ bending and librational excitations in liquid $\mathrm{H}_{2} \mathrm{O}$. Chem. Phys. Lett. 404, 389-393 (2005).

15. Ramasesha, K., DeMarco, L., Mandal, A. \& Tokmakoff, A. Water vibrations have strongly mixed intra- and intermolecular character. Nat. Chem. $\mathbf{5}$, 935-940 (2013).

16. Carpenter, W. B., Fournier, J. A., Biswas, R., Voth, G. A. \& Tokmakoff, A. Delocalization and stretch-bend mixing of the $\mathrm{HOH}$ bend in liquid water. $J$. Chem. Phys. 147, 084503 (2017).

17. Marco, L. et al. Anharmonic exciton dynamics and energy dissipation in liquid water from two- dimensional infrared spectroscopy water from twodimensional infrared spectroscopy. J. Chem. Phys. 145, 094501 (2017).

18. Lindner, J. et al. Vibrational relaxation of pure liquid water. Chem. Phys. Lett. 421, 329-333 (2006).

19. Lock, A. J. \& Bakker, H. J. Temperature dependence of vibrational relaxation in liquid $\mathrm{H}_{2} \mathrm{O}$. J. Chem. Phys. 117, 1708-1713 (2002).

20. Ashihara, S., Huse, N., Espagne, A., Nibbering, E. T. J. \& Elsaesser, T. Ultrafast structural dynamics of water induced by dissipation of vibrational energy. $J$. Phys. Chem. A 111, 743-746 (2007).

21. Pakoulev, A., Wang, Z., Pang, Y. \& Dlott, D. D. Vibrational energy relaxation pathways of water. Chem. Phys. Lett. 380, 404-410 (2003).

22. Chuntonov, L., Kumar, R. \& Kuroda, D. G. Non-linear infrared spectroscopy of the water bending mode: direct experimental evidence of hydration shell reorganization? Phys. Chem. Chem. Phys. 16, 13172-13181 (2014).

23. Imoto, S., Xantheas, S. S. \& Saito, S. Ultrafast dynamics of liquid water: energy relaxation and transfer processes of the $\mathrm{OH}$ stretch and the $\mathrm{HOH}$ bend. J. Phys. Chem. B 119, 11068-11078 (2015).

24. Rey, R., Ingrosso, F., Elsaesser, T. \& Hynes, J. T. Pathways for $\mathrm{H}_{2} \mathrm{O}$ bend vibrational relaxation in liquid water. J. Phys. Chem. A 113, 8949-8962 (2009).

25. Ahmed, M., Namboodiri, V., Singh, A. K. \& Mondal, J. A. On the intermolecular vibrational coupling, hydrogen bonding, and librational freedom of water in the hydration shell of mono- and bivalent anions. J. Chem. Phys. 141, 164708 (2014).

26. Ingrosso, F., Rey, R., Elsaesser, T. \& Hynes, J. T. Ultrafast energy transfer from the intramolecular bending vibration to librations in liquid water. J. Phys. Chem. A 113, 6657-6665 (2009)

27. Kraemer, D. et al. Temperature dependence of the two-dimensional infrared spectrum of liquid $\mathrm{H}_{2} \mathrm{O}$. Proc. Natl Acad. Sci. USA 105, 437-442 (2008).

28. Nagata, Y., Yoshimune, S., Hsieh, C., Hunger, J. \& Bonn, M. Ultrafast vibrational dynamics of water disentangled by reverse nonequilibrium ab initio molecular dynamics simulations. Phys. Rev. X 5, 021002 (2015).

29. Wang, Z., Pakoulev, A., Pang, Y. \& Dlott, D. D. Vibrational substructure in the OH stretching transition of water and HOD. J. Phys. Chem. A 108, 9054-9063 (2004)

30. Torii, H. Ultrafast anisotropy decay of coherent excitations and the noncoincidence effect for delocalized vibrational modes in liquids. Chem. Phys. Lett. 323, 382-388 (2000).

31. Logan, D. E. The non-coincidence effect in the raman spectra of polar liquids Chem. Phys. 103, 215-225 (1986).

32. Ni, Y. \& Skinner, J. L. IR and SFG vibrational spectroscopy of the water bend in the bulk liquid and at the liquid-vapor interface, respectively. J. Chem. Phys. 143, $014502(2015)$ 
33. Seki, T. et al. Decoding the molecular water structure at complex interfaces through surface-specific spectroscopy of the water bending mode. Phys. Chem. Chem. Phys. 22, 10934-10940 (2020).

34. Vinaykin, M. \& Benderskii, A. V. Vibrational sum-frequency spectrum of the water bend at the air/water interface. J. Phys. Chem. Lett. 3, 3348-3352 (2012).

35. Piatkowski, L., Eisenthal, K. B. \& Bakker, H. J. Ultrafast intermolecular energy transfer in heavy water. Phys. Chem. Chem. Phys. 11, 9033-9038 (2009).

36. Rahman, A. \& Stillinger, F. H. Molecular dynamics study of liquid water. J. Chem. Phys. 55, 3336-3359 (1971).

37. Laage, D. \& Hynes, J. T. A molecular jump mechanism of water reorientation. Science 311, 832-835 (2006).

38. Laage, D., Stirnemann, G., Sterpone, F., Rey, R. \& Hynes, J. T. Reorientation and allied dynamics in water and aqueous solutions. Annu. Rev. Phys. Chem. 62, 395-416 (2011).

39. Wernet, P. et al. The structure of the first coordination shell in liquid water. Science 304, 995-999 (2004).

40. Kühne, T. D. \& Khaliullin, R. Z. Electronic signature of the instantaneous asymmetry in the first coordination shell of liquid water. Nat. Commun. 4, 1450 (2013).

41. Fecko, C. J., Loparo, J. J., Roberts, S. T. \& Tokmakoff, A. Local hydrogen bonding dynamics and collective reorganization in water: ultrafast infrared spectroscopy of HOD/D 2 O. J. Chem. Phys. 122, 054506 (2005).

42. Lawrence, C. P. \& Skinner, J. L. Vibrational spectroscopy of HOD in liquid III. Spectral diffusion, and hydrogen- bonding and rotational dynamics. J. Chem. Phys. 118, 264-272 (2003).

43. Woutersen, S. \& Bakker, H. J. Hydrogen bond in liquid water as a brownian oscillator hydrogen bond in liquid water as a brownian oscillator. Phys. Rev. Lett. 83, 2077-2080 (1999).

44. Nienhuys, H.-K., Santen, R. Avan \& Bakker, H. J. Orientational relaxation of liquid water molecules as an activated process. J. Chem. Phys. 112, 8487 (2000).

45. Bieze, T. W. N., van derMaarel, J. R. C. \& Leyte, J. C. The intramolecular OH bond length of water in a concentrated poly(ethyleneoxide) solution. An NMR relaxation study. Chem. Phys. Lett. 216, 56-62 (1993).

46. Struis, R. P. W. J., DeBleijser, J. \& Leyte, J. C. Dynamic behavior and some of the molecular properties of water molecules in pure water and in magnesium chloride solutions. J. Phys. Chem. 91, 1639-1645 (1987).

47. van derMaarel, J. R. C., Lankhorst, D., deBleijser, J. \& Leyte, J. C. On the single-molecule dynamics of water from proton, deuterium and oxygen-17 nuclear magnetic relaxation. Chem. Phys. Lett. 122, 541-544 (1985).

48. Ludwig, R., Weinhold, F. \& Farrar, T. C. Experimental and theoretical determination of the temperature dependence of deuteron and oxygen quadrupole coupling constants of liquid water. J. Chem. Phys. 103, 6941-6950 (1995).

49. Larouche, P., Max, J.-J. \& Chapados, C. Isotope effects in liquid water by infrared spectroscopy. II. Factor analysis of the temperature effect on $\mathrm{H}_{2} \mathrm{O}$ and $\mathrm{D}_{2}$ O. J. Chem. Phys. 129, 064503 (2008).

50. Hu, Q., Zhao, H. \& Ouyang, S. Understanding water structure from Raman spectra of isotopic substitution $\mathrm{H}_{2} \mathrm{O} / \mathrm{D}_{2} \mathrm{O}$ up to 573 K. Phys. Chem. Chem. Phys. 19, 21540-21547 (2017).

51. Cowan, M. L. et al. Ultrafast memory loss and energy redistribution in the hydrogen bond network of liquid $\mathrm{H}_{2} \mathrm{O}$. Nature 434, 199-202 (2005).

52. Van DerPost, S. T. et al. Strong frequency dependence of vibrational relaxation in bulk and surface water reveals sub-picosecond structural heterogeneity. Nat. Commun. 6, 8384 (2015).

53. Hasegawa, T. \& Tanimura, Y. A polarizable water model for intramolecular and intermolecular vibrational spectroscopies. J. Phys. Chem. B 115, 5545-5553 (2011).
54. Zhong, K. et al. Vibrational mode frequency correction of liquid water in density functional theory molecular dynamics simulations with van der Waals correction. Phys. Chem. Chem. Phys. 22, 12785-12793 (2020).

\section{Acknowledgements}

We acknowledge the financial support from the European Research Council (ERC) under the European Union's Horizon 2020 research and innovation program (grant agreement no 714691), DAAD (grant agreement no 57526761), and from the MaxWater Initiative of the Max Planck Society. We thank to Sho Imoto, Jiyu Xu, and Seonchoel Cha for fruitful discussion.

\section{Author contributions}

Y.N. and J.H. conceived the research idea. C.-C.Y. and K.-Y.C. conducted the IR and pump-probe IR experiments, and M.O. conducted the Raman and hyper-Raman experiments. C.-C.Y., K.-Y.C., and J.H. analyzed the experimental data. Y.N. and T.O. carried out the simulations and analyzed the simulation data. C.-C.Y., K.-Y.C., M.O., T.S., T.O., X.Y., V.K., H.H., M.B., J.H., and Y.N. discussed and interpreted the results. C.-C.Y., K.-Y.C., M.B., J.H., and Y.N. wrote the manuscript.

\section{Funding}

Open Access funding enabled and organized by Projekt DEAL.

\section{Competing interests}

The authors declare no competing interests.

\section{Additional information}

Supplementary information is available for this paper at https://doi.org/10.1038/s41467 020-19759-w.

Correspondence and requests for materials should be addressed to M.O., J.H. or Y.N.

Peer review information Nature Communications thanks the anonymous reviewers for their contribution to the peer review of this work. Peer reviewer reports are available.

Reprints and permission information is available at http://www.nature.com/reprints

Publisher's note Springer Nature remains neutral with regard to jurisdictional claims in published maps and institutional affiliations.

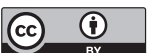

Open Access This article is licensed under a Creative Common Attribution 4.0 International License, which permits use, sharing, adaptation, distribution and reproduction in any medium or format, as long as you give appropriate credit to the original author(s) and the source, provide a link to the Creative Commons license, and indicate if changes were made. The images or other third party material in this article are included in the article's Creative Commons license, unles indicated otherwise in a credit line to the material. If material is not included in the article's Creative Commons license and your intended use is not permitted by statutory regulation or exceeds the permitted use, you will need to obtain permission directly from the copyright holder. To view a copy of this license, visit http://creativecommons.org/ licenses/by/4.0/.

(c) The Author(s) 2020 